\title{
Errors in the statistical justification for the "credibility ceiling" remain uncorrected
}

\author{
Maya B. Mathur*1 and Tyler J. VanderWeele ${ }^{2}$ \\ ${ }^{1}$ Quantitative Sciences Unit, Stanford University \\ ${ }^{2}$ Department of Epidemiology, Harvard University
}

We thank Dr. Ioannidis for his response [1] to our critiques of the "credibility ceiling" method for meta-analyses [2]. We certainly agree on a few general points: to paraphrase, all sensitivity analyses should be interpreted responsibly and with appropriate circumspection; numerous biases, not just confounding, simultaneously affect the scientific literature; and no single observational study can provide $100 \%$ certainty. We also acknowledge that the original paper introducing the credibility ceiling focuses mostly on the ceiling's effect on the "statistical significance" of the meta-analytic estimate rather than the estimate itself [3]. Yet the original paper as well as Dr. Ioannidis' subsequent work do also present meta-analytic estimates for various choices of credibility ceilings, which seems to invite readers to interpret these estimates as meaningful [3, 4. Furthermore, Dr. Ioannidis' subsequent papers specifically describe the method as "re-estimat[ing] the meta-analysis pooled effect size" [5] and even provide Stata code to "estimate the summary effect with different ceilings" 4]. We also showed that, with a fixed amount of bias in a given study, there is no limit to the actual credibility as the effect size gets larger, but we acknowledge that if, for example, the bias scales proportionately with the effect size (which seemed to be implicitly suggested in Dr. Ioannidis' comments), then the credibility would indeed be bounded by a "ceiling" of sorts. However, as explained below, our critique in no way depends upon this point, and even with these general conceptual points of agreement, the crux of our critique remains entirely unaddressed as follows.

Dr. Ioannidis reiterates his central claim: The credibility ceiling method "practically says that "no matter how large the study is, I am not willing to accept more than $(1-c) \%$

${ }^{*}$ Correspondence to: Maya B. Mathur (mmathur@stanford.edu), Quantitative Sciences Unit, 1701 Page Mill Road, Palo Alto, CA, 94304. 
that the effect is in the direction that is proposed by the observed data" ". The crux of our argument was a direct mathematical refutation of this interpretation. We demonstrated that the credibility ceiling does not yield this conclusion, because the statistical setup of the method simply does not correspond to capping at $(1-c) \%$ the probability that the true effect is in the same direction as the observed estimate. Dr. Ioannidis' response shifts the focus to a generic expression of skepticism about the state of the scientific literature and about statistical methods to address biases, and in doing so entirely sidesteps responding to the crux of our argument. We would therefore invite Dr. Ioannidis to defend his claim above about the interpretation of the credibility ceiling by demonstrating the specific flaw in our mathematical argument or by providing a correct mathematical proof that directly justifies his claim.

We had presented two thought experiments that laid bare the incorrect conclusions that the credibility ceiling can yield as a direct consequence of its incorrect statistical framework. These examples still stand. The first example, in which the uncorrected point estimate is 1, holds without loss of generality for any uncorrected point estimate (e.g., 5), and in any case, we had in mind that the effect sizes were on a scale such as the mean difference scale or the log-risk ratio, such that 1 did not represent the null. Our key points in presenting the examples were: (1) the credibility ceiling fails to change the point estimate at all in the first example, purely as an artifact of the studies' identical point estimates; and (2) the credibility ceiling shifts the point estimate in the wrong direction in the second example. Again, these points hold for any size of pooled point estimate, including those for which it is certainly not "absurd" to apply the credibility ceiling method. Dr. Ioannidis claims that the method is intended primarily to increase "skepticism" about the meta-analysis, presumably by making inference (e.g., confidence intervals and $p$-values) more conservative. But what is the statistical meaning of artificially inflated confidence intervals built around a point estimate that has been adjusted incorrectly, potentially even in the wrong direction? Unfortunately, no relevant statistical justification of inference adjusted via the credibility ceiling has ever 
been provided.

More broadly, Dr. Ioannidis dismisses the literature on sensitivity analysis for various forms of bias as "largely spurious" and "utopian" and describes our own methods for conducting sensitivity analysis for unmeasured confounding as "flawed". It is certainly true that most methods for sensitivity analysis, including our own, are exploratory and will generally not yield definitive conclusions. Hence, we agree that the methods are not perfect. However, we do believe that methodologists have a responsibility to clearly state any assumptions on which their methods rely and to provide a precise deductive argument that establishes the intended interpretation of the methods as a logical conclusion of these premises (though the evidence from the application of the methods will always be inductive). These practices are of course standard in the statistical literature, and we have followed them when proposing our own sensitivity analyses [6, 7]. We invite Dr. Ioannidis to substantiate his claim that our own methods are "flawed" by directly rebutting the mathematical arguments in our papers [6. 7]. It is rather incongruous to dismiss as "spurious" an entire statistical literature on sensitivity analysis, with little more than nebulous skepticism to support these claims, and then to propose an alternative method whose statistical framework demonstrably fails, on objective mathematical grounds, to support its intended interpretation. Again, our claim to the latter effect could, if false, be persuasively refuted if Dr. Ioannidis were to identify inaccuracies in our mathematical argument or to offer his own proof justifying his claims regarding the credibility ceiling. Ultimately, unless such a rebuttal of our actual critique is provided, we stand by our recommendation to avoid using the credibility ceiling method.

We are, however, sympathetic to what seem to be the underlying aims of the credibility ceiling approach, which we take to be examining the sensitivity of conclusions to one or more studies with potentially very large point estimates, or to one or more studies with very large sample sizes and very small variances, which could dominate the meta-analytic results. We believe that principled methods to examine such sensitivity would be useful to develop. However, as explained above, we do not think the credibility ceiling approach provides such. 
Further methodological development to do so would be valuable.

\section{REFERENCES}

[1] JPA Ioannidis. Using credibility ceilings to explore skepticism about observational evidence. Under review.

[2] MB Mathur and TJ VanderWeele. Fundamental problems with the "credibility ceiling" method for meta-analyses. Under review.

[3] Georgia Salanti and John PA Ioannidis. Synthesis of observational studies should consider credibility ceilings. Journal of Clinical Epidemiology, 62(2):115-122, 2009.

[4] Stefania I Papatheodorou, Konstantinos K Tsilidis, Evangelos Evangelou, and John PA Ioannidis. Application of credibility ceilings probes the robustness of meta-analyses of biomarkers and cancer risk. Journal of Clinical Epidemiology, 68(2):163-174, 2015.

[5] Leandro Fórnias Machado de Rezende, Thiago Hérick de Sá, Georgios Markozannes, Juan Pablo Rey-López, I-Min Lee, Konstantinos K Tsilidis, John PA Ioannidis, and José Eluf-Neto. Physical activity and cancer: An umbrella review of the literature including 22 major anatomical sites and 770000 cancer cases. Br J Sports Med, 52(13):826-833, 2018.

[6] Peng Ding and Tyler J VanderWeele. Sensitivity analysis without assumptions. Epidemiology, 27(3):368, 2016.

[7] Maya B Mathur and Tyler J VanderWeele. Sensitivity analysis for unmeasured confounding in meta-analyses. Journal of the American Statistical Association, 115:163-172, 2019. 